\section{Optisol vs Dexsol as storage media for preservation of human corneal epithelium}

A Greenbaum¹, SM Hasany² and D Rootman²

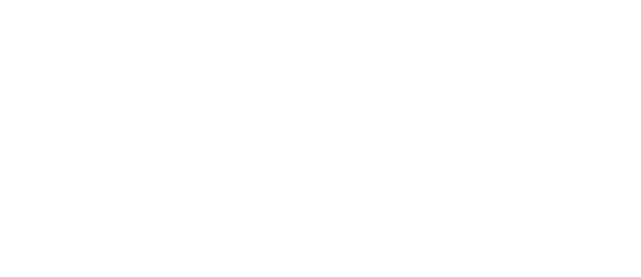

\section{Abstract}

Purpose To compare the efficacy of two storage media, Optisol GS and Dexsol, in preservation of donor corneal epithelium. Methods A total of 12 pairs of corneas not suitable for transplantation, all with intact epithelium, were used in this study, with one cornea of the pair stored in Optisol GS and its other counterpart in Dexsol. At each of three durations of storage $-1,2$, and 4 days - four of these paired corneas were prepared for light microscopy and scanning and transmission electron microscopy. Another four pairs of control cornea were prepared in the same way and placed in universal fixative.

Main outcome measures Evaluation of the corneas was made by two observers masked as to the identity of the storage medium and length of storage. Loss of epithelial cells was evaluated by light microscopy. The attachment of the epithelium to the basement membrane, cellular integrity, intercellular junctions, and intracellular organelles were evaluated and compared by electron microscopy.

Results The magnitude of epithelial loss correlated with the length of storage time. Control corneas maintained normal epithelium with preservation of all epithelial cell layers. Corneas stored for $\mathbf{1}$ day had minimal damage of the epithelium. Corneas stored for 2 days had a slight increase in epithelial damage, and corneas stored for up to 4 days showed a marked increase in epithelial damage. There were no significant differences between the two storage media. The basal cell layer was maintained in both the media at all time points, usually in good condition with mild-to-moderate damage in some cases.

Conclusions Loss of donor epithelium is related mainly to the length of storage and is similar in both Optisol GS and Dexsol. The storage time should be less than 4 days, especially when performing penetrating keratoplasty on patients with ocular surface disorders.

Eye (2004) 18, 519-524. doi:10.1038/sj.eye.6700693

Keywords: optisol; dexsol; storage media; corneal transplantation; graft survival; keratoplasty

\section{Introduction}

The integrity of the corneal epithelium is essential for graft survival following penetrating keratoplasty. Loss of donor corneal epithelium exposes uncovered stroma until migration of the host epithelium re-covers the surface. When re-epithelization does not occur, a persistent epithelial defect develops, which may lead to infection, scarring, vascularization, thinning, perforation, and failure of graft. Healthy, proliferative donor corneal epithelium has been shown to persist long after transplantation and contribute to the repair of epithelial defect. The transplantation of an intact epithelium is particularly important for graft survival in patients with ocular surface disease such as severe dry eyes, Stevens-Johnson syndrome, ocular pemphigoid, chemical burn, and limbal deficiency.

Despite the important role of epithelium, little attention has been paid to its fate during corneal storage for transplantation. Various corneal preservation media have been compared clinically and a number of methods have been used to assess endothelial viability. Comparable data for corneal epithelium are virtually nonexistent.

Optisol GS and Dexsol are two chondroitinsulphate-based, commercial storage media available in North America (Table 1). They differ mainly in the concentration of chondroitin
${ }^{1}$ Goldschleger Eye Institute Sheba Medical Center Tel-Hashomer and Sackler Faculty of Medicine Tel Aviv University Israel

${ }^{2}$ University of Toronto Department of Ophthalmology The Toronto Hospital and Eye Bank of Canada (Ontario Division) Toronto, Canada

Correspondence:

A Greenbaum

Goldschleger Eye Institute Sheba Medical Center Tel-Hashomer 52621 Israel

Tel: + 97235302874

Fax: + 97235302822

E-mail: aron-g@

zahav.net.il

Received: 30 December 2002

Accepted in revised form: 25 July 2003

The authors have no financial interest related to the product of this manuscript. 
Table 1 Constituents of two corneal storage media

\begin{tabular}{lll}
\hline Constituent & Optisol & Dexsol \\
\hline Base medium & $\begin{array}{l}\text { Hybrid of TC-199 } \\
\text { and MEM }\end{array}$ & MEM \\
Chondroitin sulphate, (\%) & 2.5 & 1.35 \\
Dextran, (\%) & 1 & 1 \\
HEPES buffer & Yes & Yes \\
Gentamycin sulphate & Yes & Yes \\
Streptomycin & Yes & No \\
Nonessential amino acids & 0.1 & 0.1 \\
(mmol/l) & & \\
Sodium pyruvate (mmol/l) & 1 & 1 \\
Additional antioxidants & Yes & Yes \\
Sodium bicarbonate & Yes & Yes \\
\hline
\end{tabular}

MEM=minimum essential medium; TC-199=tissue culture medium 199; HEPES=N-2-hydroxyethylpiperazine- $\mathrm{N}^{\prime}$-2-ethane sulfphonic acid.

Table 2 Data of 16 pairs of corneas, 12 stored in Optisol and Dexsol storage medium, and four control pairs in a universal fixative

\begin{tabular}{lcccl}
\hline $\begin{array}{l}\text { Age of } \\
\text { donor } \\
\text { (years) }\end{array}$ & $\begin{array}{c}\text { Time from } \\
\text { death to } \\
\text { enucleation } \\
(h)\end{array}$ & $\begin{array}{c}\text { Time from } \\
\text { enucleation to } \\
\text { preservation } \\
(h)\end{array}$ & $\begin{array}{c}\text { Storage } \\
\text { time } \\
\text { (days) }\end{array}$ & \\
\hline 77 & 1 & 11 & 0 & Cause of death \\
74 & 7 & 9 & 0 & Myocardial infarction \\
68 & 4 & 16 & 0 & Lung cancer \\
74 & 3 & 19 & 0 & Pulmonary Oedema \\
73 & 1 & 20 & 1 & Cerebrovascular accident \\
74 & 1 & 21 & 1 & Pneumonia \\
74 & 3 & 13 & 1 & Cerebrovascular accident \\
63 & 6 & 19 & 1 & Aortic aneurysm rupture \\
76 & 2 & 5 & 2 & Cancer of colon \\
79 & 3 & 17 & 2 & Cancer of colon \\
77 & 1 & 9 & 2 & Myoinfarction \\
62 & 6 & 20 & 2 & Cancer of bladder \\
76 & 2 & 9 & 4 & Myocardial infarction \\
75 & 1 & 19 & 4 & Myocardial infarction \\
72 & 1 & 18 & 4 & Myocardial infarction \\
61 & 2 & 7 & 4 & Congestive heart failure \\
\hline
\end{tabular}

sulphate, which is $2.5 \%$ in Optisol and $1.35 \%$ in Dexsol, and the addition of multiple components (vitamins, hydroxyproline, and ATP precursors) to the Optisol solution. ${ }^{1}$ Optisol has been advertised as a superior medium and there are anecdotal reports that it might be better for epithelial preservation. We carried out an assessment of the effect of these widely used preparations on the corneal epithelium.

\section{Methods}

The study was carried out on 16 pairs of human corneas from 16 donors (Table 2) provided by the Eye Bank of
Table 3 Characteristics of donor corneas stored in Optisol and Dexsol vs control corneas

\begin{tabular}{lll}
\hline & Optisol and Dexsol & Control \\
\hline No. of corneas & 12 pairs & 4 pairs \\
Donor age (years) & $71.83 \pm 6.22$ & $73.25 \pm 3.77$ \\
& $(61-79)$ & $(68-77)$ \\
Time of death to & $2.41 \pm 1.83$ & $3.75 \pm 2.5$ \\
enucleation (h) & $(1-6)$ & $(1-7)$ \\
Time from enucleation to & $14.50 \pm 5.39$ & $13.75 \pm 4.57$ \\
preservation (h) & & \\
& $(1-21)$ & $(9-19)$ \\
\hline
\end{tabular}

Values expressed as means $\pm S D$, range in parentheses.

Canada and obtained from whole eyes that were kept in a moist chamber at $4{ }^{\circ} \mathrm{C}$. Only epithelial-intact corneal pairs were used, as determined by slit-lamp examination before corneoscleral rim excision, and again immediately following placement in a preservation medium (12 pairs) or universal fixative (four pairs) prior to refrigeration. No corneas had an epithelial defect at the time of immersion in medium. Donor corneas were harvested according to standard EBAA protocol. The corneoscleral rims were dissected from whole globes that had been decontaminated in $1 \%$ povidine iodine solution and rinsed in normal saline solution.

One cornea from each of 12 pairs of corneas was stored in Optisol GS (Chiron Intraoptics, Irvine, CA, USA) and its other counterpart in Dexsol (Chiron Ophthamics, Irvine, CA, USA), both at $4^{\circ} \mathrm{C}$. The other four paired corneas were processed in the same way and placed in a universal fixative after decontamination without being placed in storage media; these corneas served as control.

Donor information including age of donor, time from death to enucleation, time from enucleation to storage, and causes of death were recorded in the eye bank and are summarized in Tables 2 and 3. Four paired corneas from each storage media were randomly selected at 1, 2, and 4 days and examined in the slit-lamp biomicroscope for epithelial integrity and fixed in a universal fixative. After gross inspection, each cornea was divided into three parts - one half and two quarters: the half was used for scanning electron microscopy, one quarter for light microscopy, and one quarter for transmission electron microscopy. Photographs were taken of each specimen at low, intermediate, and high magnifications to evaluate cell integrity and intracellular organelles. The four pairs of control corneas were evaluated by the same technique.

The corneal light microscopy specimens and transmission electron microscopy photographs were evaluated by two experienced examiners masked as to the identity of the storage medium and duration of 
storage. A rating scale was used based on the number of cell layers, cellular integrity, attachment of epithelium to basement membrane, intercellular junctions, and intracellular organelles.

An attempt to evaluate epithelial status by scanning electron microscopy failed when superficial dead cells obscured our view of the deeper layers in the stored corneas (Figure 1).

\section{Results}

Since all donor corneas were paired, the mean $( \pm S D)$ donor age ${ }^{*}(71.83 \pm 6.22$ years $)$, time from death to enucleation $(2.41 \pm 1.83 \mathrm{~h})$, time from enucleation to preservation in storage medium $(14.50 \pm 5.39 \mathrm{~h})$, and time in storage medium were the same (Table 3 ).

Slit-lamp examination performed prior to and immediately after placement in storage medium showed that none of the donor corneas had an epithelial defect. Although slit-lamp examination immediately before fixation also indicated epithelial cell integrity, the structural studies disclosed changes in the epithelial cells of tissue stored in both the tested media.

There were no significant differences between the epithelium of the corneas stored in Optisol GS and Dexsol in the number of cell layers, distinction of cell shapes, and presence of intracellular organelles at any of the measurement intervals. There was a definite relationship between the duration of storage and increasing extent of epithelial damage.

Cellular integrity was maintained in the control donor corneas, as evidenced by light microscopy and transmission electron microscopy (Figure 2). Moderate intercellular oedema was apparent and the basal cells were flat or cuboidal rather than columnar. The superficial layer of epithelium was smooth, with preserved microvilli and microplicae. Normal mitochondria, endoplasmic reticula, and nuclei were found in all epithelial cells except in sloughing epithelial cells. The epithelial basement membrane appeared normal.

In contrast, all corneas kept in storage medium showed progressive epithelial damage at each interval, beginning 1 day after storage. The superficial cells exhibited mild separation and some began to fall off. Some remaining epithelial cells were flattened, and all epithelial cells were tightly adherent with a normal appearance of the cytoplasm and nucleus.

By day 2, both Optisol GS- and Dexsol-stored corneas exhibited less defined cell shapes and borders. Sloughing of the external medium and epithelial cell layers was observed. The epithelium was reduced in thickness and was composed generally of two to three cell layers. The basal cell layer of both stored tissues appeared normal
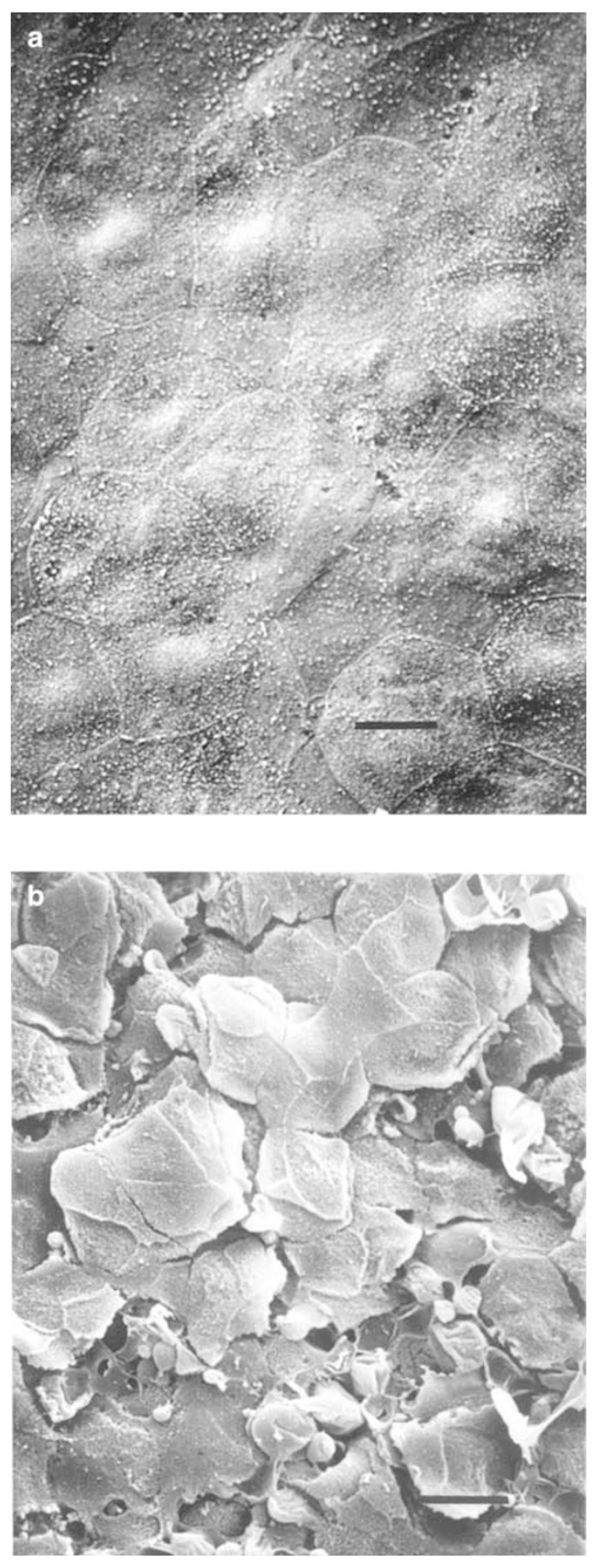

Figure 1 Scanning electron micrograph of the donor corneal epithelium. (a) Control specimen processed for microscopy without being placed in storage media. Normal superficial epithelial cells, closely packed with defined borders. The outer surface shows numerous microvilli. (b) Corneal epithelium stored for 2 days in Dexsol storage media. There is marked cellular disruption, folded cell membrane, and cellular debris. The scale bar represents $11 \mu \mathrm{m}$. 


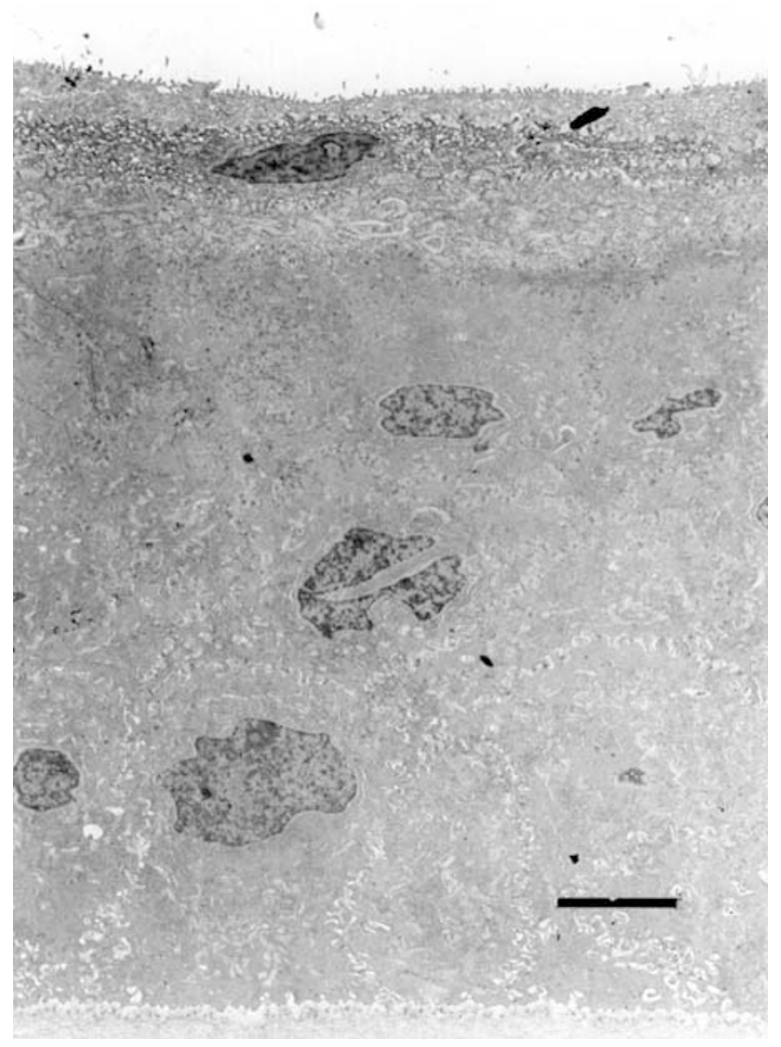

Figure 2 Transmission electron micrograph of the human corneal epithelium from donor corneas prepared without storage (control) showing normal corneal epithelium with preservation of all five cell layers. The interdigitation between cells and microvilli are also preserved. The scale bar represents $5 \mu \mathrm{m}$.

with intact cell junctions and preserved intracellular organelles; this layer remained attached to the basement membrane (Figure 3).

At 4 days of storage, corneas from both the storage media had lost all superficial epithelial cell layers and were left with only a basal cell layer, which in some sections showed a mild separation from the basement membrane (Figure 4). Some deep squamous cells sent projections between two adjacent basal cells, reaching the basal membrane and even separating the basal surface of the cell from the basement membrane (Figure 5). The basal cells did, however, contain normal cellular organelles.

\section{Discussion}

Although previous research has compared endothelial cell preservation after Optisol and Dexsol storage, epithelial cell preservation has not been addressed. In a clinical trial comparing the two media, ${ }^{2}$ endothelial cell loss and clinical outcome were the same, indicating that
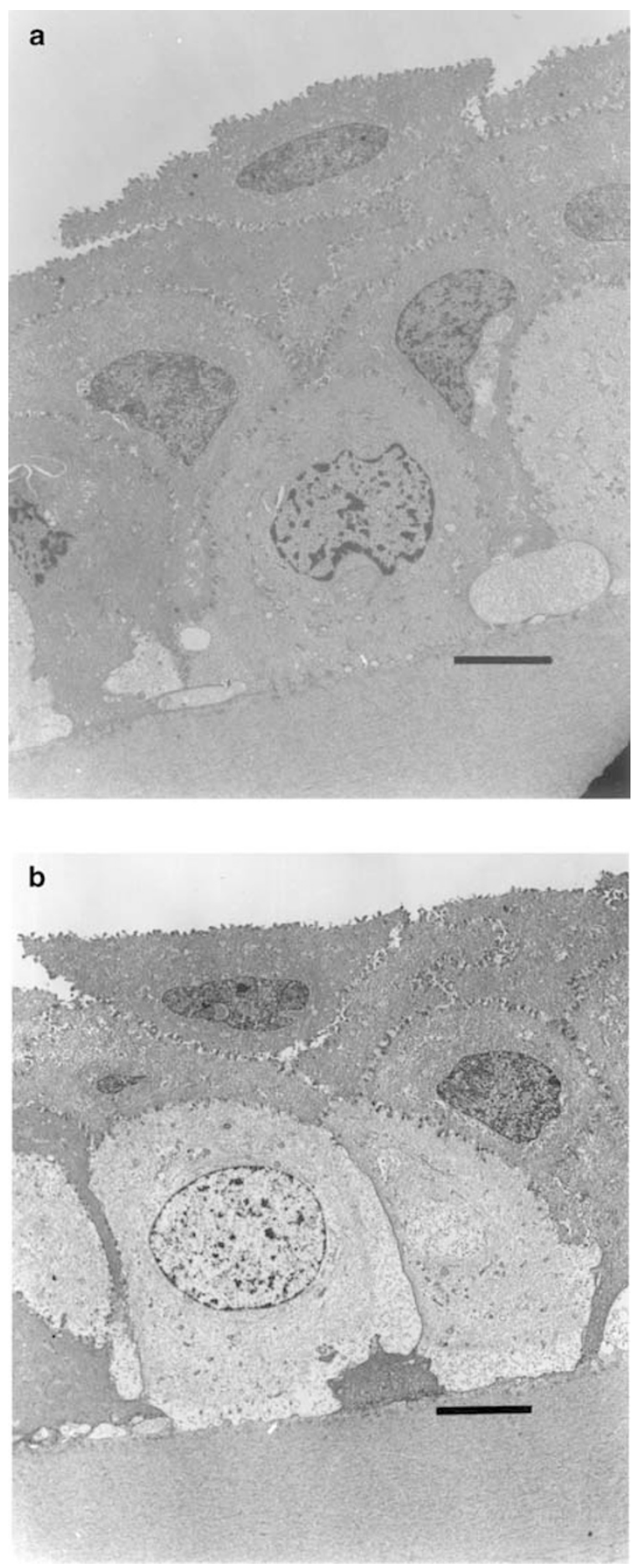

Figure 3 Transmission electron micrograph of the human corneal epithelium from paired donor corneas after 2 days in Optisol (a) or Dexsol (b). The donor age was 76 years. There is loss of superficial cells with damage to the intermediate layer, while the structure of the basal cells is normal in both the specimens. The scale bar represents $5 \mu \mathrm{m}$. 

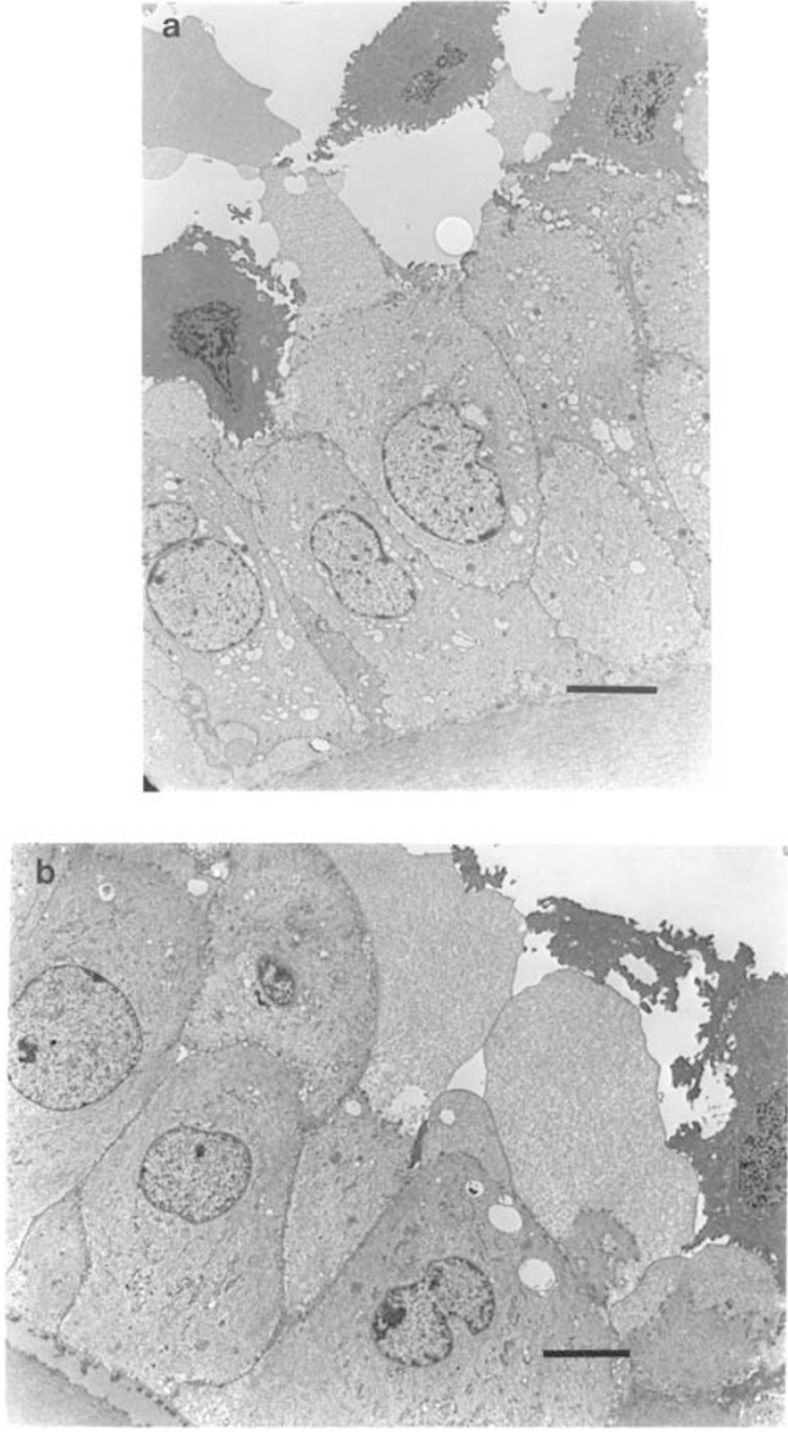

Figure 4 Transmission electron micrograph of the human corneal epithelium from paired donor corneas after 4 days of storage in Optisol (a) or Dexsol (b). The donor age was 72 years. Loss of the external and medium epithelial cell layers can be observed in both the specimens. The remaining basal epithelial cells display a flat appearance without damage. The scale bar represents $5 \mu \mathrm{m}$.

clinically Optisol is no more effective than Dexsol in preserving the corneal endothelium. Another in vitro study reported thinner corneal tissue and better preservation of structural morphology of endothelium during Optisol storage. ${ }^{3}$ Although the viability of the donor endothelium is far more important than the epithelium for the success of penetrating keratoplasty, the increasing use of donor corneal epithelium in cases of severe disturbances of epithelial integrity dictates a reexamination of storage effect on the epithelial layer.

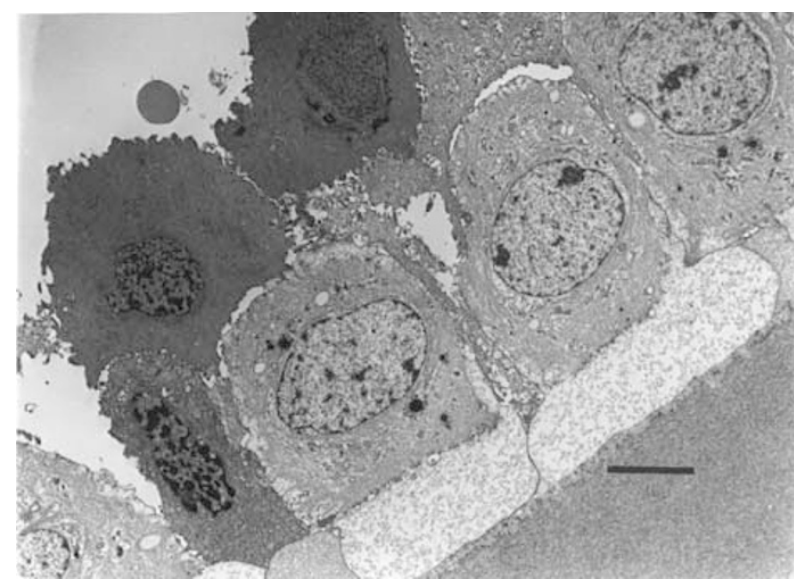

Figure 5 Transmission electron micrograph of donor cornea after 4 days in Optisol storage medium. Extensions of squamous cells have apparently reached the basal membrane and separated it from the adjacent basal cells. These extensions contain a granular material, probably cytoplasmic debris of the squamous cell. The scale bar represents $5 \mu \mathrm{m}$.

We found no significant difference in the epithelial integrity and cell loss between the two storage media. Our data demonstrate progressive loss of epithelial cells during corneal preservation. Indeed, after 4 days of storage nearly all the cells were lost, with the exception of the basal cell layer that was intact in most sections and exhibited mild separation in a few sections. Although only the basal cells remained of the epithelium after 4 days, these cells contained normal organelles and had retained their ability to control their intracellular volume, indicating that metabolic pumps were probably active. ${ }^{4}$ Basal cells in the corneal epithelium are known to play a crucial role in the maintenance and regeneration of corneal epithelial cell layers. ${ }^{5,6}$ Since they are thought to be transient amplifying cells that are rapid cycling and can amplify cell mass effectively by limited rounds of mitosis, ${ }^{7}$ the clinical significance of being left with only the basal cell layer remains to be elucidated.

An interesting observation of a yet unknown significance was the presence of projections emanating from deep squamous epithelial cells. These projections were observed between basal cells and even extending between the basal cells and the basement membrane of the epithelium. It is not clear whether this is an active process or the result of degeneration of the basal cells, which would mean the basal cells were undergoing apoptosis and losing all capacity for proliferation.

Other investigators have also examined epithelial integrity during corneal storage. A previous study on KSol-preserved corneas showed a progressive breakdown of the epithelial cells after 4 days of storage, ${ }^{8}$ and 
reported that the epithelial integrity was unpredictable during 4 and 20 days of storage. In a recent experimental study, Means et $a l^{9}$ found the retention of epithelial cells for at least 6 days after storage in Optisol GS media. While our results demonstrated severe epithelial loss in both the storage media after 4 days, our average donor age $(71.83 \pm 6.22$ vs $57.8 \pm 2.3$ years $)$ and enucleation to preservation times $(14.50 \pm 5.39$ vs $2.66 \pm 0.26 \mathrm{~h})$ exceeded those of Means et al and could contribute to our different results. Our observation is consistent, however, with that of Kim et $a l,{ }^{10}$ who found an association between the degree of epithelial defect 1 day after penetrating keratoplasty and increased storage time in Optisol GS medium. These authors found a high probability of an epithelial defect 1 day postpenetrating keratoplasty for corneas stored in Optisol GS for 4 days.

In summary, according to our study Optisol appears to be no more effective than Dexsol in preserving the integrity of human corneal epithelium as evaluated by electron microscopy. The finding of progressive loss of epithelial cells during storage in both the media studied over 4 days indicates that fresher corneal tissue is necessary to prevent the loss of donor epithelium, especially in cases where maintaining the epithelial integrity and viability is crucial for long-term graft function and survival. The search should continue for the ideal corneal storage medium that is economical, safe, and assures minimal epithelial cell loss.

\section{References}

1 Doughman DJ. Tissue storage. In: Krachmer JH, Mannis MJ, Holland EJ (eds). Cornea, Vol 1, Chapter 39. Mosby: St Louis, MO, 1997, pp. 510-511.

2 Lass JH, Bourne WM, Musch DC, Sugar A, Gordon JF, Reinhart WJ et al. A randomized, prospective doublemasked clinical trial of Optisol vs Dexsol corneal storage media. Arch Ophthalmol 1992; 110: 1404-1408.

3 Lindstrom RL, Kaufman HE, Skelnik DL, Laing RA, Lass JH, Musch DC et al. Optisol corneal storage medium. Am J Ophthalmol 1992; 114: 345-356.

4 Trump BF, Ginn FL. The pathogenesis of subcellular reaction to lethal injury. In: Bajusz E, Jasmin G (eds). Methods and Achievements in Experimental Pathology, Examples of Descriptive and Functional Morphology, Vol 4. S Karger AG: Basel; 1969, pp 1-29.

5 Lamprecht J. Symmetric and asymmetric cell division in rat corneal epithelium. Cell Tissue Kinet 1990; 23: 203-216.

6 Sharma A, Coles WH. Kinetics of corneal epithelial maintenance and graft loss. Invest Ophthalmol Vis Sci 1989; 30: 1962-1971.

7 Tseng SCG. Concept and application of limbal stem cells. Eye 1989; 3: 141-157.

8 Farge EJ, Font RA, Wellhelmus KR, Gilbert ML, Mehta RS and Kretzer FL. Morphologic changes of K-sol preserved human corneas. Cornea 1989; 8: 159-169.

9 Means LT, Geroski DH, Hernault N, Grossniklaus HE, Kim T and Edelhauser HF. The corneal epithelium after Optisol-GS storage. Cornea 1996; 15: 599-605.

10 Kim T, Palay DA, Lynn M. Donor factors associated with epithelial defect after penetrating keratoplasty. Cornea 1996; 15: 451-456. 